\title{
Zerebrovaskuläre Ereignisse, Kopfschmerzen und Livedo racemosa - Blickdiagnose?
}

\section{Cerebrovascular events, headache and livedo racemosa - diagnosis at a glance?}

Stefan Krämer', Georg Richter², Uwe Goldammer', Eckhart Sindern'

\author{
Autoren \\ Institute \\ 1 Neurologie, DIAKOVERE Friederikenstift \\ 2 Institut für Pathologie Richter \\ Schlüsselwörter \\ Sneddon-Syndrom, Antiphospholipid-Antikörper-Syndrom, \\ Thrombophilie \\ Key words \\ thrombophilia, Sneddon's syndrome, antiphospholipid \\ antibody syndrome \\ eingereicht 16.08 .2019 \\ akzeptiert $\quad 04.10 .2019$ \\ Bibliografie \\ DOI https://doi.org/10.1055/a-1028-7132 \\ Fortschr Neurol Psychiatr 2020; 88: 263-265 \\ (c) Georg Thieme Verlag KG Stuttgart · New York \\ ISSN 0720-4299 \\ Korrespondenzadresse \\ Dr. Stefan Krämer \\ Neurologie DIAKOVERE Friederikenstift \\ Humboldstraße 5 \\ 30169 Hannover \\ Tel.: 05111292321 \\ Fax: 05111292404 \\ E-Mail: stefan.kraemer@diakovere.de
}

\section{ZUSAMMENFASSUNG}

Das Sneddon-Syndrom ist eine seltene Erkrankung, gekennzeichnet durch zerebrovaskuläre Ereignisse und Livedo racemosa. Häufig bestehen autoimmunologische Komorbiditäten, insbesondere das Antiphospholipid-Antikörper-Syndrom. Die Pathophysiologie ist weiterhin nicht vollständig geklärt. Eine kausale Therapie existiert nicht. Der berichtete Fall zeigt eine Patientin mit einer thrombophilen Form des Sneddon-Syndroms mit den Leitsymptomen Kopfschmerz und thromboembolische Ereignisse. Es werden die Symptome, Laborparameter, Histologie und Differentialdiagnosen erläutert.

\begin{abstract}
Sneddon's syndrome is a rare disease characterized by cerebrovascular events and livedo racemosa. There are often autoimmunological comorbidities, especially antiphospholipid antibody syndrome. The underlying pathophysiology is still not fully clarified. A causal therapy does not exist. The reported case shows a patient with a thrombophilic form of Sneddon's syndrome with the main symptoms of headache and thromboembolic events. Symptoms, laboratory parameters, histology and differential diagnoses are explained.
\end{abstract}

\section{Einleitung}

Das Sneddon-Syndrom ist eine seltene Erkrankung, die durch eine nichtinflammatorische thrombotische Vaskulopathie zu zerebrovaskulären Ereignissen und der charakteristischen Livedo racemosa führt. Livedo racemosa ist eine Hautveränderung, gekennzeichnet durch eine verzweigte Struktur livider Verfärbungen mit dem Erscheinungsbild unterbrochener Kreise [1]. Erstbeschrieben wurde dieses Erkrankungsbild durch Kimming im Jahr 1959 [2]. Namensgeber war lan Bruce Sneddon im Jahr 1965 [3]. Die Inzidenz wird mit 1:1000000 angegeben [4]. Die Ätiologie der
Erkrankung ist weitestgehend ungeklärt, es zeigen sich jedoch in etwa der Hälfte der Fälle autoimmunologische Komorbiditäten [5]. Unterschieden werden in der Literatur drei Klassifikationen des Syndroms: eine autoimmunologische Form, bei der entweder ein Antiphospholipid-Antikörper-Syndrom oder ein systemischer Lupus erythematodes oder eine Lupus-ähnliche Erkrankung vorliegen, eine Form mit Thrombophilie und ein primäres Sneddon-Syndrom [6]. Pathophysiologisch werden eine Endarteriits obliterans und eine Proliferation der glatten Muskelzellen der Gefäßwand beschrieben [7]. 
Fall

Die Patientin stellte sich im Alter von 23 Jahren aufgrund chronisch rezidivierender frontaler drückender Cephalgien in unserer Klinik vor. Sie beschrieb zudem eine passagere Hypästhesie des linken Armes einige Monate zuvor. In der Vorgeschichte waren mehrfache Beinvenenthrombosen sowie eine Lungenarterienembolie und rezidivierende Aborte beschrieben. Zudem bestand eine leichte Depression. Die neurologische Untersuchung erbrachte keine Auffälligkeiten. Der Blutdruck lag bei Aufnahme bei $190 / 100 \mathrm{mmHg}$. Inspektorisch fielen am Rumpf sowie an Oberarmen und Oberschenkeln livide Hautverfärbungen auf, die auch bei Wärme persistent waren und sich als unterbrochene Kreise darstellten (s. D Abb. 1). In der kranialen Kernspintomographie zeigten sich einige fleckförmige periventrikulär gelegene Läsionen der weißen Substanz mit teilweisem Nachweis von Hämosiderinablagerungen als Hinweis auf stattgehabte Mikroblutungen. In der digitalen Subtraktions-Angiographie (DSA) wurden keine Kaliberschwankungen der Gefäße nachgewiesen. Das Blutbild wies eine Thrombozytopenie von etwa 80.000/ $\mu$ l auf. Das weitere Routinelabor zeigte keine Auffälligkeiten, insbesondere keine beeinträchtigte Nierenfunktion. Die immunologische Diagnostik erbrachte einen leicht erhöhten Titer antinukleärer Antikörper (ANA) mit 1:640 (Norm < 1:160) bei negativen antineutrophilen zytoplasmatischen Antikörpern (ANCA). Die Antiphospholipid-Antikörper waren negativ. Die Blutkörperchensenkungsgeschwindigkeit (BSG) lag in mehreren Untersuchungen zwischen 22 und $84 \mathrm{~mm}$ nach einer Stunde (Norm < 10 mm), das C-reaktive-Protein (CRP) war nicht erhöht. Liquordiagnostisch konnte eine leichte lymphozytäre Pleozytose (9 Zellen pro Mikroliter) mit einem Albuminquotienten von 9,2 festgestellt werden. Eine periphere Neuropathie wurde elektrophysiologisch nicht nachgewiesen. Der histopathologische Befund der Hautbiopsie erbrachte den Nachweis erweiterter und teils stenosierter Blutgefäße mit dem Bild einer
Proliferation von Endothelzellen und einer zum Teil verdickten Gefäßwand entsprechend einer nichtvaskulitischen okklusiven Arteriopathie ( $\triangleright$ Abb. 2).

\section{Diskussion}

Der vorliegende Fall vereint Symptome verschiedener Organsysteme. Es besteht eine Beteiligung von zentralem Nervensystem mit Nachweis von postischämischen und posthämorrhagischen zerebralen Läsionen und Kopfschmerzen, eine arterielle Hypertonie, eine Thrombozytopenie, Thrombophilie sowie Hautveränderungen.

Kopfschmerzen sind bei Patienten mit Sneddon-Syndrom die häufigste klinische Präsentation [8]. Eine arterielle Hypertonie zeigt sich bei betroffenen Patienten ebenfalls häufig, beschrieben sind bis zu 65\% [9]. Die Hautveränderungen der Patientin sind als Livedo racemosa zu charakterisieren, da sie die typischen morphologischen Merkmale aufweisen. Sie bestehen im Gegensatz zur physiologischen Livedo reticularis dauerhaft und sind nicht durch Kälteexposition hervorrufbar. Der Befund der Hautbiopsie zeigt nach der Einteilung von Zelger et al. [10] ein Stadium zwischen Grad I mit „Endotheliitis“ und Grad II mit Obstruktion der kleinen und mittleren subkutanen Blutgefäße. Der Zusammenhang dieser Befunde wäre pathophysiologisch durch eine Mikroangiopathie zu erklären. Die autoimmunologische Diagnostik und das klinische Bild sind nicht vereinbar mit einer typischen Vaskulitis. Die Diagnose einer thrombozytopenischen Mikroangiopathie (TMA) ist bei fehlender Niereninsuffizienz unwahrscheinlich. Die Anamnese der Patientin mit rezidivierenden Thrombosen und Aborten lässt an ein Antiphospholipid-Syndrom (aPL-Syndrom) denken. In der Literatur sind diverse thrombophile Komorbiditäten bei Patienten mit Sneddon-Syndrom dokumentiert, etwa eine Protein-C- oder Protein-S-Resistenz, erhöhtes Thromboglobulin, veränderte Plasminogenverhältnisse oder eine familiäre Anti-

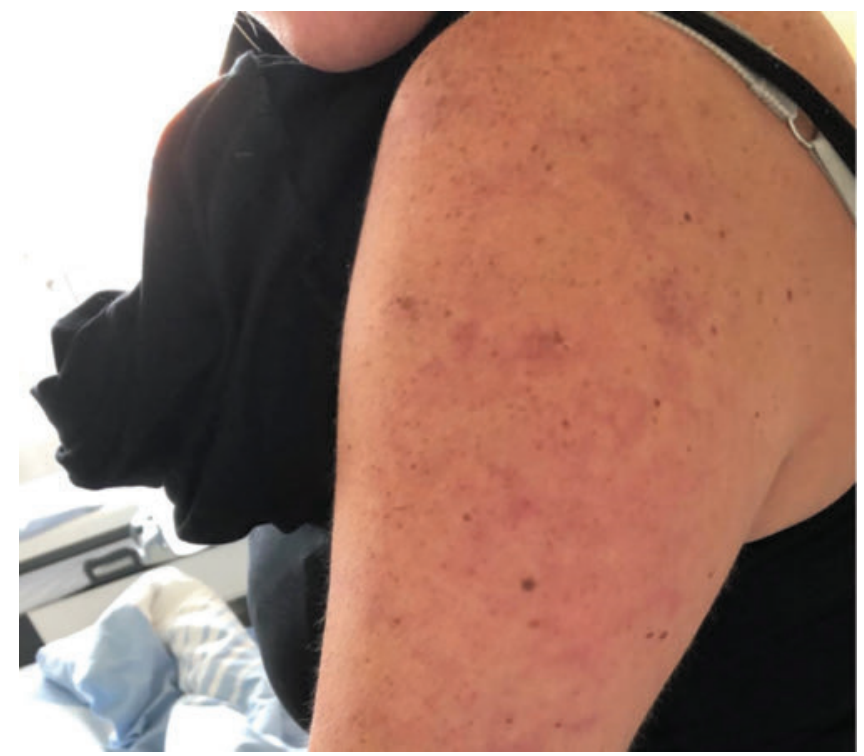

- Abb. 1 Oberarm der Patientin mit dem Bild einer Livedo racemosa.

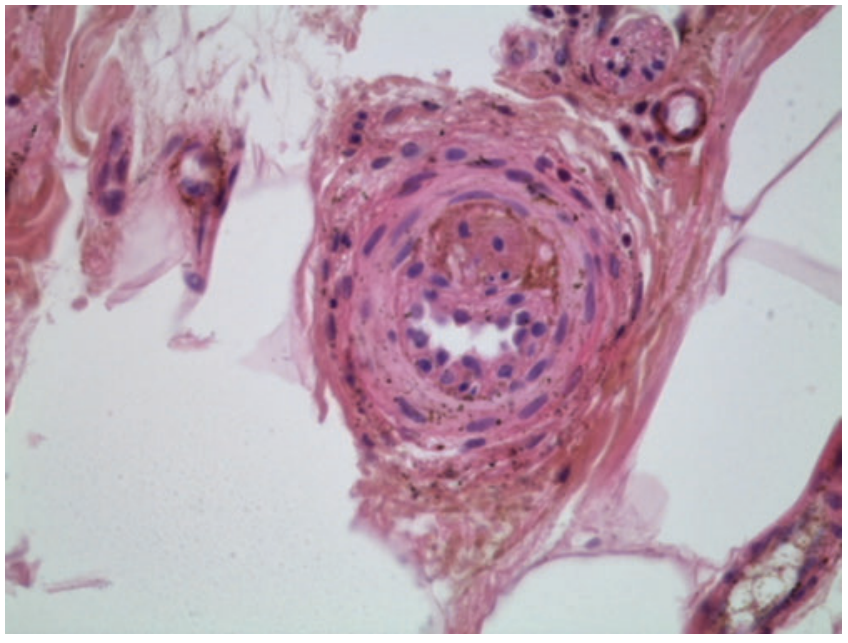

- Abb. 2 Hautbiopsie in HE Färbung: Proliferation von Endothelzellen und eine zum Teil verdickte Gefäßwand. 
thrombin-III-Resistenz [11]. Die wiederholte Bestimmung der aPL-Antikörper bei der Patientin ergab negative Befunde. Der Nachweis von aPL-Antikörpern bei Sneddon-Syndrom ist zwischen 0 und $82 \%$ beschrieben [12]. Erhöhte ANA-Titer fanden sich in der Arbeit von Francès et al. [12] bei $11 \%$ von Patienten mit Sneddon-Syndrom ohne Nachweis von Antiphospholipid-Antikörpern (aPL) bzw. bei 27\% aPL-positiver Patienten. Eine Überschneidung von Sneddon-Syndrom und systemischem Lupus erythematodes (SLE) wird in der Literatur ebenfalls diskutiert. Man fand u.a. erstgradig Verwandte von Patienten mit Sneddon-Syndrom auch ohne aPL-Antikörper, die einen SLE aufwiesen [13]. Die Diagnose eines systemischen Lupus erythematodes (SLE) lässt sich in diesem Fall bei unzureichend erfüllten ACR-Kriterien nicht stellen, jedoch geben die erhöhte BSG und der erhöhte ANA-Titer Hinweise auf eine generalisierte Immunerkrankung. Ein Übergang hin zu einem manifesten SLE im Verlauf wäre denkbar. Therapeutisch gibt es keine klare Empfehlung bei dieser Erkrankung. Eine immunsupressive Therapie scheint nicht wirksam zu sein [14]. Khamashta et al. berichteten eine Überlegenheit von Warfarin gegenüber Aspirin in Bezug auf zerebrovaskuläre Ereignisse [15]. Im berichteten Fall wurde die Patientin mit Apixaban behandelt, zudem erfolgte eine antihypertensive und antidepressive Therapie.

Das charakteristische Bild der Livedo racemosa in Kombination mit zerebrovaskulären Ereignissen, einer Thrombophilie und der histopathologische Befund der Hautbiopsie sprechen in dem vorgestellten Fall für eine thrombophile Form des Sneddon-Syndroms.

\section{Interessenkonflikt}

Die Autoren geben an, dass kein Interessenkonflikt besteht.
Literatur

[1] Ehrmann S. Ein neues Gefäßsymptom bei Lues. Medizinische Wochenschrift 1907; 777-782

[2] Kimmig J. Arteriolopathie: Livedo racemosa. Dermatologische Wochenschrift 1959; 139: 211

[3] IB S. Cerebro-Vascular Lesions and Livedo Reticularis. Br J Dermatol 1965; 77: 180-185

[4] Zelger B, Sepp N, Stockhammer G et al. Sneddon's syndrome. A longterm follow-up of 21 patients. Arch Dermatol 1993; 129: 437-447

[5] Kalashnikova La NE, Kushekbaeva A. Gracheva La Anticardiolipin antibodies in Sneddon's syndrome. Neurology 1990; 40: 464-467

[6] Tourbah A, Piette JC, Iba-Zizen MT et al. The naturalcourse of cerebral lesions in Sneddon syndrome. Arch Neurol 1997; 54: 53-60

[7] Marsch Wc MR. Generalized racemose livedo with cerebrovascular lesions (Sneddon syndrome): An occlusive arteriolopathy due to proliferation and migration of medial smooth muscle cells. $\mathrm{Br} J$ Dermatol 1985; 112: 703-708

[8] Tietjen GE, Al Qasmi MM, Shukairy MS. Livedo reticularis and migraine: Amarker for stroke risk? Headache 2002; 42: 352-355

[9] Schellong SM, Weissenborn K, Niedermeyer J et al. Classification of Sneddon's syndrome. Vasa 1997; 26: 215-221

[10] Zelger B, Sepp N, Schmid KW et al. Life history of cutaneous vascular lesions in Sneddon's syndrome. Hum Pathol 1992; 23: 668-675

[11] Wu S, Xu Z, Liang H. Sneddon's syndrome: A comprehensive review of the literature. Orphanet Journal of Rare Diseases 2014; 9: 215

[12] Francès $C$, Papo $T$, Wechsler $B$ et al. Sneddon syndrome with or without antiphospholipid antibodies. A comparative study in 46 patients. Medicine (Baltimore) 1999; 78: 209-219

[13] Francès C, Piette JC. The mystery of Sneddon syndrome: Relationship with antiphospholipid syndrome and systemic lupus erythematosus. J Autoimmun 2000 Sep; 15 (2): 139-143

[14] Akbal A, Kurtaran A, Selcuk B et al. A rarely seen syndrome in rehabilitation of hemiplegia: Antiphospholipid antibody-negative Sneddon's syndrome. Rheumatol Int 2010; 30: 1489-1491

[15] Khamashta MA, Cuadrado M], Mujic F et al. The management of thrombosis in the antiphospholipid-antibody syndrome. N Engl ] Med 1995; 332: 993-997 